\title{
Research Article \\ Optimal Premium Pricing for a Heterogeneous Portfolio of Insurance Risks
}

\author{
Athanasios A. Pantelous, Nicholas E. Frangos, \\ and Alexandros A. Zimbidis
}

Department of Statistics, Athens University of Economics and Business, 76 Patision Street, Athens 104 34, Greece

Correspondence should be addressed to Athanasios A. Pantelous, a.pantelous@liverpool.ac.uk

Received 20 March 2009; Accepted 11 June 2009

Recommended by Ričardas Zitikis

The paper revisits the classical problem of premium rating within a heterogeneous portfolio of insurance risks using a continuous stochastic control framework. The portfolio is divided into several classes where each class interacts with the others. The risks are modelled dynamically by the means of a Brownian motion. This dynamic approach is also transferred to the design of the premium process. The premium is not constant but equals the drift of the Brownian motion plus a controlled percentage of the respective volatility. The optimal controller for the premium is obtained using advanced optimization techniques, and it is finally shown that the respective pricing strategy follows a more balanced development compared with the traditional premium approaches.

Copyright (C) 2009 Athanasios A. Pantelous et al. This is an open access article distributed under the Creative Commons Attribution License, which permits unrestricted use, distribution, and reproduction in any medium, provided the original work is properly cited.

\section{Introduction}

In this paper, we develop a stochastic control model for a heterogeneous portfolio system with $k$ classes of insured risks, each containing a large number of insured units. The basic pricing principle suggests that each policyholder should pay a premium proportional to the risk that imposes to the total pool. Bowers et al. [1] suggest a time-constant premium, $\pi_{i}=\left(1+\theta_{i}\right) \mu_{i}$, for each class $i$, where $\mu_{i}$ is the expected value of the total risk of class $i$, and $\theta_{i}$ is the loading factor that is determined such that the probability that the total expected claims of class $i$ exceed the respective premiums paid equals to $\xi_{i}$. Quite recently, Zaks et al. [2] proposed an optimal premium calculation for each class of risks by minimizing the expected squared distance between the total claim amount and the total premium income in each class. 
Here, we consider this problem in a more general framework by letting also some kind of solvency interaction between the different classes and using the tools of stochastic control theory. Although optimal control theory was developed by engineers in order to investigate the properties of dynamic systems of difference or differential equations, it has also been successfully applied to financial and actuarial problems. Tustin [3] was one of the firsts who spot a possible analogy between the industrial and engineering processes and postwar macroeconomic policy-making (see Holly and Hallett, [4], for further historical details). In the insurance context, Borch [5] firstly identified the potential synergy between actuarial problems and control processes. Martin-löf [6] proposed a certain pricing model using the tools of control theory. Thereafter, a series of papers in this area have been also produced, see for instance, Asmussen and Taksar [7], Schäl [8], Højgaard and Taksar [9], Taksar [10], Hipp and Taksar [11], Irgens and Paulsen [12], Yang and Zhang [13], and so forth.

A brief outline of the paper is as follows. Section 2 provides the incentives and the typical modelling features of the problem. Moreover, it is devoted to some standard results of stochastic control theory. Section 3 provides the approximation solution for the matrix Riccati differential equation. In Section 4, we provide an interesting practical example with three classes containing several independent identically distributed (i.i.d.) insurance risks. Some interesting and insightful diagrams are also available, while Section 5 concludes the whole paper.

\section{The Framework Heterogeneous Risk Model}

We consider an insurance heterogeneous portfolio composed of $k$ risk classes under the following conditions.

(a) Class $i$ contains $n_{i}$ risks, that is, $X_{i, 1}, X_{i, 2}, \ldots X_{i, n_{i}}$, for $i=1,2, \ldots, k$ and $n=\sum_{i=1}^{k} n_{i}$. Each risk in the $i$ th-class is driven by an independent standard Brownian motion $(\mathrm{sBm})$ and can take positive or negative sign. It is positive when the insurance company pays a claim or negative when the company recovers an amount of money (e.g., due to fraud claims). This uncertainty is modeled via a probability space, $(\Omega, \mathcal{F}, D)$. The flow of information is given by the natural filtration $\{\mathcal{F}\}_{t \in[0, T]}$; that is, the $P$-augmentation of a one-dimensional Brownian filtration. Without loss of generality we assume that $\{\mathcal{F}\}_{t \in[0, T]}=\mathcal{F}$, that is, the observable events are all eventually known. So, we have the following system of stochastic differential equations:

$$
d X_{i, l}(t)=m_{i, l}(t) d t+\sigma_{i, l}(t) d W_{t}^{i, l}
$$

for $l=1,2, \ldots, n_{i}$ and $i=1,2, \ldots, k$.

(b) All sizes $n_{i}, i=1,2, \ldots, k$ are large enough to determine the deterministic functions $m_{i, l}(t) \in L^{2}\{[0, T) ; \mathbb{R}\}$, and $\sigma_{i, l}(t) \in L^{2}\{[0, T) ; \mathbb{R}\}$, which represent the drift and the volatility, respectively, of the specific $l$ risk in the $i$ class for $l=1,2, \ldots, n_{i}$ and $i=1,2, \ldots, k$. Consequently, the total risk $S_{i}=\sum_{l=1}^{n_{i}} X_{i, l}$ of the $i$ th class, obeys the following system:

$$
d S_{i}(t)=\sum_{l=1}^{n_{i}} m_{i, l}(t) d t+\sum_{l=1}^{n_{i}} \sigma_{i, l}(t) d W_{t}^{i, l}, \quad i=1,2, \ldots, k
$$


(c) For each class $i$, the total premium $\pi_{i}(t)$ is calculated according to the ordinary differential equation (2.3):

$$
d \pi_{i}(t)=\sum_{l=1}^{n_{i}} m_{i, l}(t) d t+\sum_{l=1}^{n_{i}} \varepsilon_{i, l}(t) \sigma_{i, l}(t) d t, \quad i=1,2, \ldots, k,
$$

where $\varepsilon_{i, l}(t)$ is the "loading factor" at time $t$ for the specific $l$ risk in the $i$ class of risks, $i=1,2, \ldots, k$. The $\varepsilon_{i, l}(t) \in L^{2}\{[0, T) ; \mathbb{R}\}$ is the controller for the appropriate premium pricing strategy which is determined instantly, at every time $t$.

(d) The accumulated profit/loss of the total portfolio $\Pi(t)$ at time $t$ is derived as

$$
\Pi(t)=\sum_{i=1}^{k} \Pi_{i}(t)
$$

where $\Pi_{i}(t)$ is the accumulated profit or loss at time $t$ for the $i$ th class of risks, $i=1,2, \ldots, k$, derived by the following differential equation:

$d \Pi_{i}(t)=($ Investment income earned from the fund in the time interval $[t, t+d t))$

$+($ Profit/Loss of theiclass of risks in the time interval $[t, t+d t))$

$+($ Solvency interaction in the time interval $[t, t+d t))$

or equivalently expressed in mathematical functions:

$$
\begin{aligned}
d \Pi_{i}(t)= & a_{i}(t) \Pi_{i}(t) d t+d \pi_{i}(t)-d S_{i}(t) \\
& +\left[\lambda_{1 i}(t) \Pi_{1}(t)+\cdots+\lambda_{i-1 i}(t) \Pi_{i-1}(t)\right. \\
& \left.+\lambda_{i i}(t) \Pi_{i}(t)+\lambda_{i+1 i}(t) \Pi_{i+1}(t)+\cdots+\lambda_{k i}(t) \Pi_{k}(t)\right] d t
\end{aligned}
$$

where $a_{i}(t)$ is the rate of return (or borrowing) for the accumulated profit (or loss) at time $t$ for the $i$ th class of risks, $i=1,2, \ldots, k$.

$\lambda_{i j}(t)$ is the percentage of the accumulated profit or loss (solvency) transferred from the $i$ th to the $j$ th class of risks at time $t: \sum_{j=1}^{k} \lambda_{i j}(t)=1$ for all $i=1,2, \ldots, k$. Or equivalently, substituting equations (2.2) and (2.3) into (2.6) we obtain

$$
\begin{aligned}
d \Pi_{i}(t)= & a_{i}(t) \Pi_{i}(t) d t+\sum_{l=1}^{n_{i}} \varepsilon_{i, l}(t) \sigma_{i, l}(t) d t-\sum_{l=1}^{n_{i}} \sigma_{i, l}(t) d W_{t}^{i, l} \\
& +\left[\lambda_{1 i}(t) \Pi_{1}(t)+\cdots+\lambda_{i-1 i}(t) \Pi_{i-1}(t)+\lambda_{i i}(t) \Pi_{i}(t)\right. \\
& \left.+\lambda_{i+1 i}(t) \Pi_{i+1}(t)+\cdots+\lambda_{k i}(t) \Pi_{k}(t)\right] d t
\end{aligned}
$$

for $i=1,2, \ldots, k$. 
Thus, we derive, in matrix form, the (nonhomogeneous) linear stochastic controlled differential equation:

$$
\begin{gathered}
d \bar{\Pi}(t)=\{A(t) \bar{\Pi}(t)+\Sigma(t) \bar{\varepsilon}(t)\} d t-\Sigma(t) d \bar{W}(t), \\
\bar{\Pi}(0)=0,
\end{gathered}
$$

where $A(t), \Sigma(t) \in L^{\infty}\left\{[0, T) ; \mathbb{R}^{k \times k}\right\}$

$$
\begin{aligned}
& A(t)=\left[\begin{array}{cccc}
a_{1}(t)+\lambda_{11}(t) & \lambda_{21}(t) & \cdots & \lambda_{k 1}(t) \\
\lambda_{12}(t) & a_{2}(t)+\lambda_{22}(t) & \cdots & \lambda_{k 2}(t) \\
\vdots & \vdots & \ddots & \vdots \\
\lambda_{1 k}(t) & \lambda_{2 k}(t) & \cdots & a_{k}(t)+\lambda_{k k}(t)
\end{array}\right] \\
& \bar{\Pi}(t)=\left[\begin{array}{c}
\Pi_{1}(t) \\
\Pi_{2}(t) \\
\vdots \\
\Pi_{k}(t)
\end{array}\right], \quad \bar{\varepsilon}(t)=\left[\begin{array}{c}
\bar{\varepsilon}_{1}(t) \\
\bar{\varepsilon}_{2}(t) \\
\vdots \\
\bar{\varepsilon}_{k}(t)
\end{array}\right], \quad \bar{\varepsilon}_{i}(t)=\left[\begin{array}{c}
\varepsilon_{i, 1}(t) \\
\varepsilon_{i, 2}(t) \\
\vdots \\
\varepsilon_{i, n_{i}}(t)
\end{array}\right], \\
& \Sigma(t)=\operatorname{diag}\left\{\sigma_{1}(t) ; \sigma_{2}(t) ; \ldots ; \sigma_{k}(t)\right\}, \quad \sigma_{i}(t)=\left[\begin{array}{llll}
\sigma_{i, 1}(t) & \sigma_{i, 2}(t) & \ldots & \sigma_{i, n_{i}}(t)
\end{array}\right], \\
& d \bar{W}(t)=\left[\begin{array}{c}
d \bar{W}_{1}(t) \\
d \bar{W}_{2}(t) \\
\vdots \\
d \bar{W}_{k}(t)
\end{array}\right], \quad d \bar{W}_{i}(t)=\left[\begin{array}{c}
d W_{i, 1}(t) \\
d W_{i, 2}(t) \\
\vdots \\
d W_{i, n_{i}}(t)
\end{array}\right] .
\end{aligned}
$$

For any $t \in[0, T]$, we denote the set of all 5-tuples $(\Omega, \mathcal{F}, p, \bar{W}(\cdot), \bar{\varepsilon}(\cdot))$ satisfying the following.

(i) $(\Omega, \mathbb{F}, P)$ is a complete probability space.

(ii) $\{\bar{W}(t)\}_{t \geq 0}$ is a $k$-dimensional standard Brownian motion defined on $(\Omega, \mathcal{F}, p)$ over $[0, T]$ (with $W(0)=0$ almost surely), and $\mathcal{F}_{t}=\sigma\{W(r): 0 \leq r \leq$ $t$ ) augmented by all the $D$-null sets in $\mathcal{F}$.

(iii) The controller $\bar{\varepsilon}(\cdot) \in L_{F}^{2}\left\{[0, T) ; \mathbb{R}^{k}\right\}$.

(iv) Under $\bar{\varepsilon}(\cdot)$, for any $\bar{\Pi}(0) \in \mathbb{R}^{k}(2.8)$ admits a unique solution $\bar{\Pi}(\cdot)$ on $\left(\Omega, \mathcal{F},\left\{\mathcal{F}_{t}\right\}_{t \geq 0}, D\right)$. 
(e) Finally, we aim to minimize the following quadratic cost criterion (under the constraint of differential equation (2.8)):

$$
J^{\bar{\varepsilon}}(t, \bar{\Pi})=\mathbb{E}^{t, \bar{\Pi}}\left\{\int_{0}^{T}\left[\bar{\Pi}^{\prime}(t) Q \bar{\Pi}(t)+\left(\bar{\varepsilon}(t)-\bar{\varepsilon}_{\tau}\right)^{\prime} R\left(\bar{\varepsilon}(t)-\bar{\varepsilon}_{\tau}\right)\right] d t+\bar{\Pi}^{\prime}(\mathrm{T}) G \bar{\Pi}(\mathrm{T})\right\},
$$

where, $T>0$ and define $Q=\operatorname{diag}\left\{\theta_{1}, \theta_{1}, \ldots, \theta_{1}\right\} \in \mathbb{R}^{n}, R=\operatorname{diag}\left\{\theta_{2}, \theta_{2}, \ldots, \theta_{2}\right\} \in \mathbb{R}^{n}$, and $G=\operatorname{diag}\left\{\left(1-\theta_{1}-\theta_{2}\right),\left(1-\theta_{1}-\theta_{2}\right), \ldots,\left(1-\theta_{1}-\theta_{2}\right)\right\} \in \mathbb{R}^{n}$ and $\theta_{1}, \theta_{2}$ are weighting factors, that is, $0 \leq \theta_{1}+\theta_{2} \leq 1$. Note that $(\cdot)^{\prime}$ is the transposed matrix of $(\cdot)$.

The weights $\theta_{1}$ and $\theta_{2}$ measure the impact that occurs when the control variables $\bar{\Pi}(t)$ and $\left(\bar{\varepsilon}(t)-\bar{\varepsilon}_{\tau}\right)$ are changed. Obviously, with $1-\theta_{1}-\theta_{2}$, we penalize the accumulated profit/loss at the end of the finite time-horizon $T$. Definitely, the weighting parameters would be obtained after research and negotiations with all parties involved in the private insurance pricing system (i.e., authorities, managerial policy of the insurance company, customers, etc).

Furthermore, we seek to obtain analytical results (formulae) rather than purely numerical ones, since our model has a very practical interest. In that direction, we propose a stochastic linear-quadratic approach for the determination of the optimal premium policy of a heterogeneous portfolio of risks. Stochastic linear quadratic (SLQ) problems have been studied by many authors, among them we merely mention Wonham [14], McLane [15], Davis [16], Ichikawa [17], Chen and Yong [18], and so forth. In many recent works on mathematical finance (see option pricing, utility optimization) as well as in engineering problems (note that, here, it is sometimes called energy cost function) this criterion has been extensively applied.

Our stochastic linear-quadratic approach allows us to nest both conventional analyses of optimal surplus stabilization policy $(\bar{\Pi}(t), t \in[0, T))$ and analyses of optimal premium smoothing policy $(\bar{\varepsilon}(t), t \in[0, T])$. Analytically, this criterion aims to a stable $\bar{\varepsilon}(t)$ and consequently stable premium policy, which is highly desirable by the customers of the insurance company. Additionally, the criterion aims to small values of the surplus fund $\bar{\Pi}(t)$ for all times $t \in[0, T)$ and especially a small value for the final fund value at time $T$. The last condition secures that insurance company will have no problems with solvency requirements (large surplus/deficit) or explosion of its surplus/deficit at the end of the control period. Finally, in practice, it should be pointed out that the $\bar{\varepsilon}_{\tau}$ has to predefine considering the policy of its insurance company. Thus, in this model the required risk managerial policy is closely followed. Actually, this very important benefit is derived from our optimal stochastic model.

The above Stochastic Linear Quadratic (SLQ) problem described by (2.8) and (2.13) at $(0, \bar{\Pi}(0)) \in[0, T] \times \mathbb{R}^{k}$ is solvable if there exists a control $\left(\Omega, \mathcal{F}, \boldsymbol{p}, W(\cdot), \bar{\varepsilon}^{*}(\cdot)\right)$ such that

$$
J\left(0, \bar{\Pi}(0) ; \bar{\varepsilon}^{*}(\cdot)\right)=\inf _{\bar{\varepsilon}(\cdot) \in U^{\omega}[0, T]} J(0, \bar{\Pi}(0) ; \bar{\varepsilon}(\cdot)) \triangleq V(0, \bar{\Pi}(0)) .
$$

In the case, where $\bar{\varepsilon}^{*}(\cdot)$ is an optimal control, the corresponding $\bar{\Pi}^{*}(\cdot)$ and $\left(\bar{\Pi}^{*}(\cdot), \bar{\varepsilon}^{*}(\cdot)\right)$ are defined as optimal state process and optimal pair, respectively, to our problem. Closing this section, we provide the basic formulae (see also the appendix). The optimal controller is given by a feedback mechanism as

$$
\bar{\varepsilon}^{*}(t)=\bar{\varepsilon}_{\tau}-\Psi(t) \bar{\Pi}(t), \quad \text { for } t \in[0, T],
$$


where $\Psi(t) \triangleq R^{-1} \Sigma^{\prime}(t) P(t)$ and $P(\cdot) \in C\left([0, T] ; \mathbb{R}^{k \times k}\right)$ is the solution of

$$
\begin{gathered}
\dot{P}(t)+P(t) A(t)+A^{\prime}(t) P(t)+Q(t)-P(t) \Sigma(t) R^{-1} \Sigma^{\prime}(t) P(t)=0, \\
P(T)=G, \quad \text { a.e. } t \in[0, T] .
\end{gathered}
$$

\section{A Special Case for the General Solution of $P(t)$}

The general solution of the Riccati type equation (2.16) is not an easy task. Here, we describe in brief the solution for $P(\cdot) \in C\left([0, T] ; \mathbb{R}^{k \times k}\right)$, which is actually symmetric. We define analytically the time-varying matrix $P(t)$ as follows:

$$
P(t)=\left(P_{i j}(t)\right)_{i, j=1,2, \ldots, k^{\prime}}
$$

where $P_{i j}(t)$ are scalars continuous functions.

In order to simplify our calculations (the full extension requires quite cumbersome calculations), we determine the matrix $A \in \mathbb{R}^{k \times k}$ to be also symmetric, that is, $\lambda_{i j}(t)=\lambda_{j i}(t)$, for $i \neq j$ and also assume the following.

(i) $a_{i}(t)=a(t)$, the same rate of return earned by the accumulated profit or loss at time $t$ for each class of risk, $i=1,2, \ldots, k$.

(ii) $\lambda_{i j}(t)=\lambda(t)$, the same percentage of profit or loss transferred from the $i$ th class of risks to the $i$ th class of risks at time $t, \lambda_{i i}(t)=1-(k-1) \lambda(t)$ for each class of risk, $i=1,2, \ldots, k$.

The expression of (2.16) can be rewritten as follows:

$$
\dot{P}(t)+A(t) P(t)+P(t) A(t)+Q(t)-P(t) \Sigma(t) R^{-1} \Sigma(t) P(t)=0,
$$

where the symmetric matrix $A(t)$ takes the following format:

$$
A(t)=\left[\begin{array}{cccc}
a(t)+(1-(k-1) \lambda(t)) & \lambda(t) & \cdots & \lambda(t) \\
\lambda(t) & a(t)+(1-(k-1) \lambda(t)) & \cdots & \lambda(t) \\
\vdots & \vdots & \ddots & \vdots \\
\lambda(t) & \lambda(t) & \cdots & a(t)+(1-(k-1) \lambda(t))
\end{array}\right] .
$$

Then, we consider the first three terms of (3.2), that is,

$$
A(t) P(t)+P(t) A(t)+Q(t)=\left(\Theta_{i j}(t)\right)_{i, j=1,2, \ldots, k^{\prime}}
$$

where, the above matrix is symmetric,

$$
(A(t) P(t)+P(t) A(t)+Q(t))^{\prime}=A(t) P(t)+P(t) A(t)+Q(t),
$$


as $A, P$, and $Q$ are $k \times k$ symmetric matrices. Thus,

$$
\begin{aligned}
\Theta_{i \leq j}= & 2[a+(1-(k-1) \lambda)] P_{i j}+\lambda\left[\sum_{l=1}^{i} P_{l i}+\sum_{l=i}^{k} P_{i l}-P_{i j}-P_{i i}\right] \\
& +\lambda\left[\sum_{l=1}^{j} P_{l j}+\sum_{l=j}^{k} P_{j l}-P_{i j}-P_{j j}\right]+\theta_{1} 1_{i=j} .
\end{aligned}
$$

We also calculate the $\Sigma(t) R^{-1} \Sigma^{\prime}(t)$, that is,

$$
\Sigma(t) R^{-1} \Sigma^{\prime}(t)=\frac{1}{\theta_{2}}\left[\begin{array}{cccc}
\sigma_{1}(t) \sigma_{1}^{\prime}(t) & 0 & \cdots & 0 \\
0 & \sigma_{2}(t) \sigma_{2}^{\prime}(t) & \cdots & 0 \\
\vdots & \vdots & \ddots & \vdots \\
0 & 0 & \cdots & \sigma_{k}(t) \sigma_{k}^{\prime}(t)
\end{array}\right]
$$

and thereafter define

$$
P(t) \Sigma(t) R^{-1} \Sigma^{\prime}(t) P(t)=\left(Z_{i j}(t)\right)_{i, j=1,2, \ldots, k}
$$

We can easily prove that $P \Sigma R^{-1} \Sigma P$ is also symmetric, as $P$ is symmetric, and $\Sigma$ and $R$ for $j=1,2, \ldots, k$ are diagonal matrices. Thus, for $i=1,2, \ldots, k$

$$
Z_{i \leq j}=\frac{1}{\theta_{2}}\left[\sum_{l=1}^{i} \sigma_{l} \sigma_{l}^{\prime} P_{l i} P_{l j}+\sum_{l=i}^{j} \sigma_{l} \sigma_{l}^{\prime} P_{i l} P_{l j}+\sum_{l=j}^{k} \sigma_{l} \sigma_{l}^{\prime} P_{i l} P_{j l}-\sigma_{i} \sigma_{i}^{\prime} P_{i i} P_{i j}-\sigma_{j} \sigma_{j}^{\prime} P_{i j} P_{j j}\right] .
$$

Substituting the expressions (3.7) and (3.8) into (3.2), we obtain the family of the following ordinary nonlinear differential equations:

$$
\begin{aligned}
\dot{P}_{i j}+2[ & a+(1-(k-1) \lambda)] P_{i j}+\lambda\left[\sum_{l=1}^{i} P_{l i}+\sum_{l=i}^{k} P_{i l}-P_{i j}-P_{i i}\right]+\lambda\left[\sum_{l=1}^{j} P_{l j}+\sum_{l=j}^{k} P_{j l}-P_{i j}-P_{j j}\right] \\
& +\theta_{1} 1_{i \equiv j}+\frac{1}{\theta_{2}}\left[\sum_{l=1}^{i} \sigma_{l} \sigma_{l}^{\prime} P_{l i} P_{l j}+\sum_{l=i}^{j} \sigma_{l} \sigma_{l}^{\prime} P_{i l} P_{l j}+\sum_{l=j}^{k} \sigma_{l} \sigma_{l}^{\prime} P_{i l} P_{j l}-\sigma_{i} \sigma_{i}^{\prime} P_{i i} P_{i j}-\sigma_{j} \sigma_{j}^{\prime} P_{i j} P_{j j}\right]=0 .
\end{aligned}
$$

The last expression (3.10) converts the nonhomogeneous matrix Riccati differential equation (2.16) into a Cauchy problem for a system of first-order differential equations, where $P(T)=G$, a.e. $t \in[0, T]$.

Consider the Cauchy problem of the first-order differential equation:

$$
\dot{P}_{r}=f_{r}\left(t, P_{i j}\right), \quad \text { for } i \leq j, i, j, r=1,2, \ldots, k,
$$


or equivalently

$$
\dot{P}=f(t, P)
$$

where

$$
P=\left(P_{11}, P_{12}, \ldots, P_{1 n}, \ldots, P_{i j}, \ldots, P_{k k}\right),
$$

and also

$$
\begin{aligned}
f(t, & \left.P_{11}, P_{12}, \ldots, P_{1 n}, \ldots, P_{i j}, \ldots, P_{k k}\right) \\
& =\left(f_{1}\left(t, P_{11}, P_{12}, \ldots, P_{1 n}, \ldots, P_{i j}, \ldots, P_{k k}\right), \ldots, f_{k}\left(t, P_{11}, P_{12}, \ldots, P_{1 n}, \ldots, P_{i j}, \ldots, P_{k k}\right)\right)
\end{aligned}
$$

with the initial condition, after a change of variable,

$$
P(t)=P(T-t)
$$

So,

$$
P_{0}=P(0)=G, \quad \text { a.e. } t \in[0, T],
$$

where $G=\theta I_{n}, \theta=1-\theta_{1}-\theta_{2}$ is a weighting factor, that is, $0 \leq \theta \leq 1-\theta_{1}-\theta_{2}$.

The method of successive approximations obtains the solution $P(T-t)$ as the limit of a sequence of functions $P^{(n)}(T-t)$ which are determined by the following recurrence formula:

$$
P^{(n)}(T-t)=P^{(0)}+\int_{T-t}^{T} f\left(r, P^{(n-1)}(T-r)\right) d r
$$

It has been shown by Petrovsky [19] that if $f(t, P)$ is continuous in a rectangle $Q \in$ $\mathbb{R}^{n+1}\left\{|t| \leq k_{1},\left|P-P_{0}\right| \leq k_{2}\right\}$, then the error of the approximate solution $P^{(n)}(T-t)$ on the interval $[0, h]$ is estimated by the inequality

$$
\varepsilon_{n}=\left|P(T-t)-P^{(n)}(T-t)\right| \leq M K^{n} \frac{(T-t)^{n+1}}{(n+1) !},
$$

where $M=\max _{(t, P) \in \mathbb{R}^{n+1}}|f(t, P)|$, and $h$ is determined by $h=\min \left(k_{1}, k_{2} / M\right)$. 


\section{A Numerical Application for a Portfolio Composed of Three Risk Classes}

We consider a special portfolio composed of three risk classes indexed from 1 to 3 . The system of equations is described as follows:

$$
\begin{gathered}
d \bar{\Pi}(t)=\{A(t) \bar{\Pi}(t)+\Sigma(t) \bar{\varepsilon}(t)\} d t-\Sigma(t) d \bar{W}(t), \\
\bar{\Pi}(0)=0,
\end{gathered}
$$

where

$$
\begin{aligned}
& A=\left[\begin{array}{ccc}
a(t)+(1-2 \lambda(t)) & \lambda(t) & \lambda(t) \\
\lambda(t) & a(t)+(1-2 \lambda(t)) & \lambda(t) \\
\lambda(t) & \lambda(t) & a(t)+(1-2 \lambda(t))
\end{array}\right], \\
& \Sigma(t)=\operatorname{diag}\left\{\sigma_{1}(t) ; \sigma_{2}(t) ; \sigma_{3}(t)\right\}, \quad \sigma_{i}(t)=\left[\begin{array}{llll}
\sigma_{i, 1}(t) & \sigma_{i, 2}(t) & \ldots & \sigma_{i, n_{i}}(t)
\end{array}\right], \\
& \bar{\Pi}(t)=\left[\begin{array}{c}
\Pi_{1}(t) \\
\Pi_{2}(t) \\
\Pi_{3}(t)
\end{array}\right], \quad \bar{\varepsilon}(t)=\left[\begin{array}{c}
\bar{\varepsilon}_{1}(t) \\
\bar{\varepsilon}_{2}(t) \\
\bar{\varepsilon}_{3}(t)
\end{array}\right], \quad \text { where } \bar{\varepsilon}_{i}(t)=\left[\begin{array}{c}
\varepsilon_{i, 1}(t) \\
\varepsilon_{i, 2}(t) \\
\vdots \\
\varepsilon_{i, n_{i}}(t)
\end{array}\right], \\
& d \bar{W}(t)=\left[\begin{array}{c}
d \bar{W}_{1}(t) \\
d \bar{W}_{2}(t) \\
d \bar{W}_{3}(t)
\end{array}\right], \quad \text { where } d \bar{W}_{i}(t)=\left[\begin{array}{c}
d W_{i, 1}(t) \\
d W_{i, 2}(t) \\
\vdots \\
d W_{i, n_{i}}(t)
\end{array}\right] \quad \text { for } i=1,2,3
\end{aligned}
$$

We also define $P(t)$ as follows:

$$
P(t)=\left(P_{i j}(t)\right)_{i, j=1,2,3^{\prime}}
$$

where $P_{i j}(t), i \leq j, i, j=1,2,3$ are scalars continuous functions and

$$
P(T)=G \Longleftrightarrow P_{11}(T)=P_{22}(T)=P_{33}(T)=\theta, \quad P_{i j}(T)=0, \quad \forall i \neq j ; i, j=1,2,3 .
$$

Moreover, we obtain

$$
\Psi(t)=R^{-1} \Sigma^{\prime} P(t)=\frac{1}{\theta_{2}}\left[\begin{array}{ccc}
\sigma_{1}^{\prime} & 0 & 0 \\
0 & \sigma_{2}^{\prime} & 0 \\
0 & 0 & \sigma_{3}^{\prime}
\end{array}\right]\left[\begin{array}{lll}
P_{11}(t) & P_{11}(t) & P_{13}(t) \\
P_{12}(t) & P_{22}(t) & P_{23}(t) \\
P_{13}(t) & P_{23}(t) & P_{33}(t)
\end{array}\right]
$$


Table 1: Application parameters. The amounts are in $\$ 10.000$

\begin{tabular}{lcccccc}
\hline Class & Population in class & \multicolumn{1}{c}{ Claim probability } & \multicolumn{1}{c}{ Mean } & St. deviation \\
\hline$k$ & $n$ & $q$ & $\mu$ & $\sigma$ & $m$ & $v$ \\
1 & 4000 & 0.05 & 0.21 & 0.0316 & 0.0105 & 0.0463 \\
2 & 2200 & 0.10 & 1 & 0.0447 & 0.1 & 0.3 \\
3 & 800 & 0.21 & 1.3 & 0.0316 & 0.273 & 0.530 \\
\hline
\end{tabular}

where, $P_{11}(t), P_{22}(t), P_{33}(t), P_{12}(t), P_{13}(t)$, and $P_{23}(t)$ can be calculated by using the successive approximation method of Picard; see Section 3. However, in the numerical application; see also next section, a time discrete approximation is applied for the derived polynomials, see expression (4.8) and the following lines.

For the calculation of $\bar{\Pi}(t)$, we follow a numerical stochastic method (one of the simplest time discrete approximations of an Itô process) named as Euler-Maruyama approximation; see Kloeden and Platen [20] for more details. We obtain the numerical calculation of the following expression:

$$
\bar{\varepsilon}^{*}(t)=\bar{\varepsilon}_{\tau}-R^{-1} \Sigma^{\prime}(t) P(t) \Phi(t)\left\{\int_{0}^{t} \Phi(s)^{-1} \Sigma(s) d s+\int_{0}^{t} \Phi(s)^{-1} \Sigma(s) d \bar{W}(s)\right\}
$$

on $t \in\left[t_{o}, T\right]$ with the initial value $\bar{\varepsilon}^{*}\left(t_{o}\right)=\bar{\varepsilon}_{\tau}$,

$$
\bar{\varepsilon}^{*}(t)=\bar{\varepsilon}_{\tau}-R^{-1} \Sigma^{\prime} P\left(t_{o}\right) \Sigma\left\{\bar{\varepsilon}_{\tau} \int_{t_{o}}^{t} d s+\int_{t_{o}}^{t} d \bar{W}(s)\right\}
$$

and for a given discretization, the Euler approximation is a continuous time stochastic process $Y=\left\{Y(t), t_{o} \leq t \leq T\right\}$ satisfying the iterative scheme

$$
Y_{n+1}=Y_{n}-R^{-1} \Sigma^{\prime} P\left(t_{n}\right) \Sigma\left\{\bar{\varepsilon}_{\tau} \Delta_{n}+\Delta W_{n}\right\}
$$

for $n=0,1,2, \ldots, N-1$ with initial condition $Y_{0}=\bar{\varepsilon}_{\tau}$, where we have written $Y_{n}=\bar{\varepsilon}^{*}\left(t_{n}\right)$ for the value of the approximation at the discretization time $t_{n}$. Furthermore, the $\Delta_{n}$ which is defined as $\Delta_{n}=t_{n+1}-t_{n}=\delta$, in the simplest equidistant case, has step size $\delta=T / N$, and additionally, it is derived that $t_{n}=t_{o}+n \delta$.

Moreover, the increments, $\Delta W_{n}=W_{t_{n+1}}-W_{t_{n}}$, are independent Gaussian random variables with mean $\mathbb{E}\left(\Delta W_{n}\right)=0$ and variance $\mathbb{E}\left[\left(\Delta W_{n}\right)^{2}\right]=\Delta_{n}$. In the application we can use a sequence of independent Gaussian pseudorandom numbers generated by one of the random number generators of MatLab.

As we have mentioned before, the first class contains $n_{1}$, the second $n_{2}$, and the third $n_{3}$ insured risks $\left(n=\sum_{i=1}^{3} n_{i}\right)$. Additionally, we have probability $q_{i}, i=1,2,3$ for a claim. For this numerical application, we apply the data set which used by Zaks et al. [2]. This data consists of 7000 policyholders, 4000 for the 1st, 2200 for the 2nd, and 800 for the 3rd risk class. The mean, $\mu_{i}$, and the variance, $\sigma_{i}^{2}$, of each class and the mean, $m_{i}=\mu_{i} q_{i}$, and the variance $s_{i}^{2}=\mu_{i}^{2} q_{i}\left(1-q_{i}\right)+\sigma_{i}^{2} q_{i}$ of the total claims of each class are presented analytically in Table 1.

Furthermore, the numerical application is subject to the following basic parameters: rate of return $a=5 \%$, the percentage of profit or loss (solvency) transferred equals to $\lambda=10 \%$, 


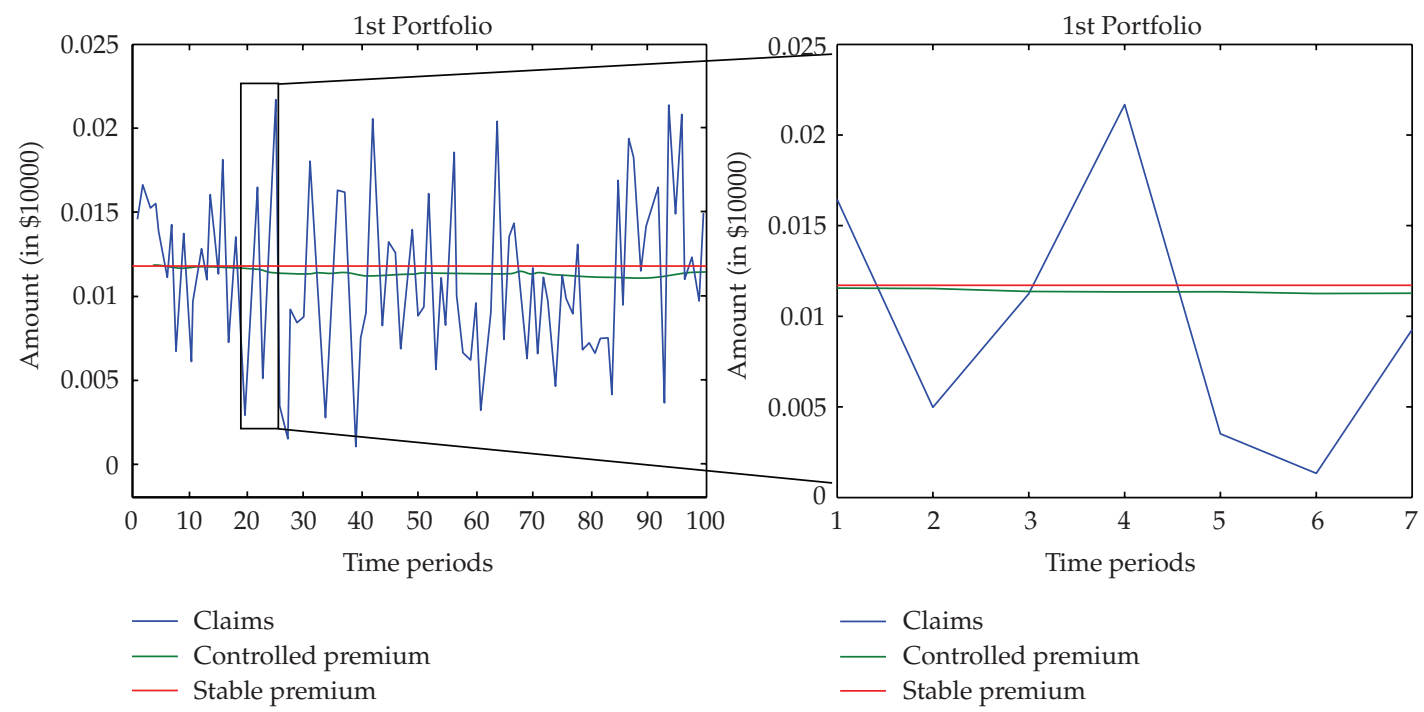

(a)

Figure 1: 1st class of a heterogeneous Portfolio, during 100 unit-time period (an enlargement region, where the results are more clear).

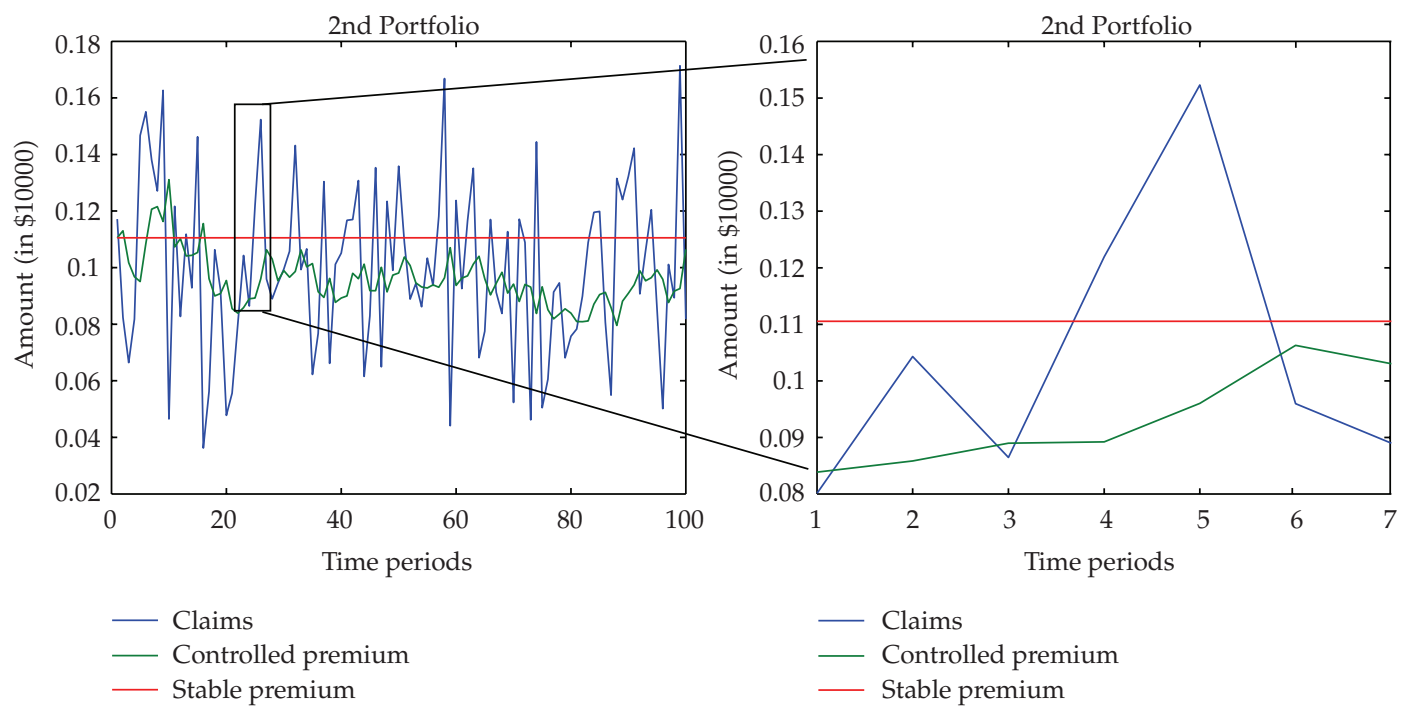

(a)

Figure 2: 2nd class of a heterogeneous Portfolio, during 100 unit-time period (an enlargement region, where the results are more clear).

the weights $\theta_{1}=\theta_{2}=\theta_{3}=1 / 3$, and the $T=100$ unit-time periods. Note that according to the renewal policy of each client (i.e., annual or six-month insurance contract) the insurance company may reconcile the unit-time period in which the premium is controlled.

It is clear from the figures, see Figures 1(a), 2(a), and 3(a), that there is a difference between the stable premium (which has been determined statistically; see Bowers et al. [1]) and the controlled premium (determined by the proposed dynamic approach). 


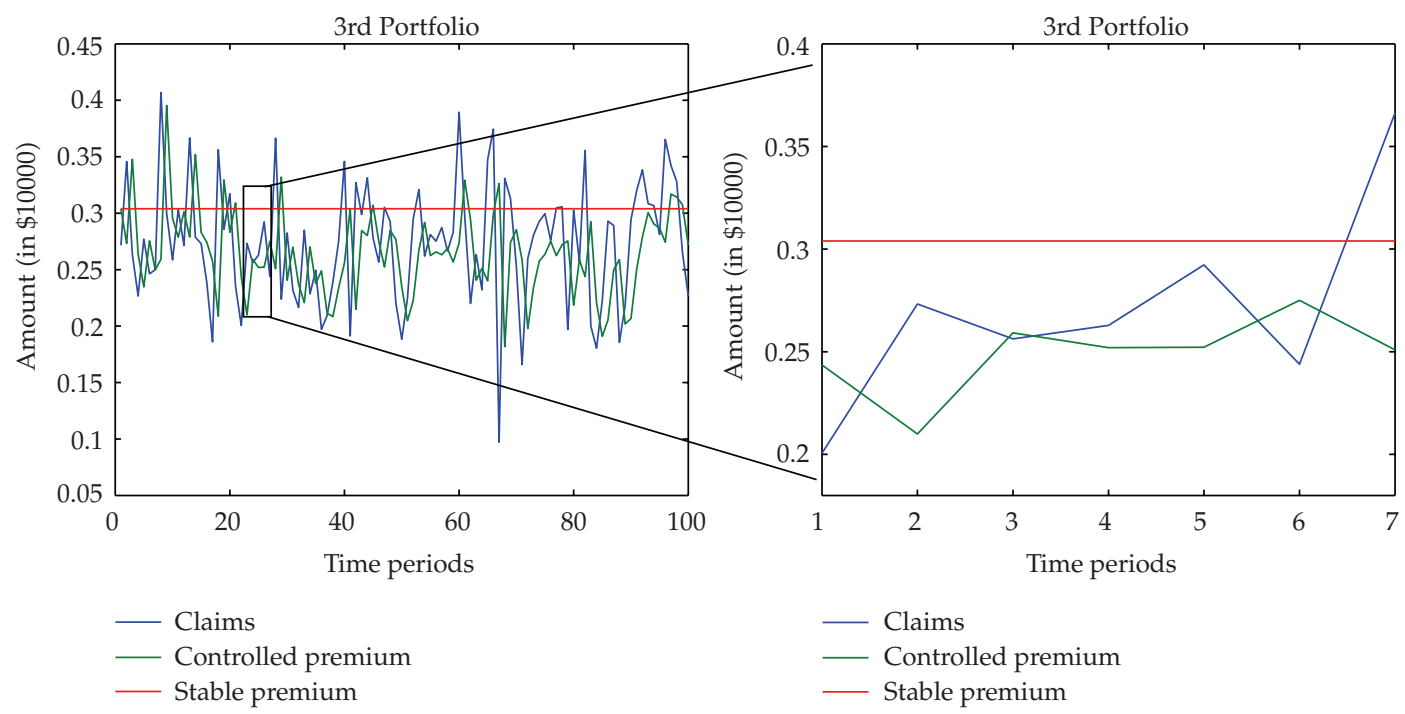

(a)

Figure 3: 3rd class of a heterogeneous Portfolio, during 100 unit-time period (an enlargement region, where the results are more clear).

Table 2: Optimal premium allocation.

\begin{tabular}{lcccc}
\hline Class & Expected principle & Uncontrolled allocation & Uniform allocation & Semiuniform allocation \\
\hline$k$ & & $\alpha_{i}=5 \%$ & $\alpha_{i}=5 \%$ & $\alpha_{i}=5 \%$ \\
1 & 109.81 & 117.08 & 116,69 & 134.05 \\
2 & 1045.81 & 1105.53 & 1110.63 & 1052.81 \\
3 & 2855.06 & 3039.18 & 3138.19 & 2875.23 \\
\hline
\end{tabular}

Following Figures $4(\mathrm{a}), 4(\mathrm{~b})$, and $4(\mathrm{c})$, we focus on the process of $\varepsilon_{i, l}(t)$, the so called "loading factor". Note that the probability that the total amount of maluses exceeds the total bonuses, for each insured class of risk, is a predetermined small number $\alpha_{i}=0.05$ for $i=1,2,3$. According to Zaks et al. [2], see Table 2, it should be stressed that the premiums of the three classes (see green line on the Figures 1(a), 2(a), and 3(a)) are smaller and so more competitive in the majority of cases (considering the uncontrolled, the uniform, and the semiuniform allocation method). This is due to the fact that the controlled premium follows more or less the evolution of the claims.

The interesting but expected result of this model is the balanced evolution of the solvency margin. The fund does not explode to infinity as the premium controller realizes the upward movement and consequently reduces the spread from the drift of the process; see Figures 5(a), 5(b), 6(a), 6(b), 7(a), and 7(b).

\section{Conclusions}

In traditional risk theory the procedure of premium calculation is well established using the classical individual risk model. Each risk $i$ is statistically described by the triplet $\left(q_{i}, \mu_{i}, \sigma_{i}^{2}\right)$ where 


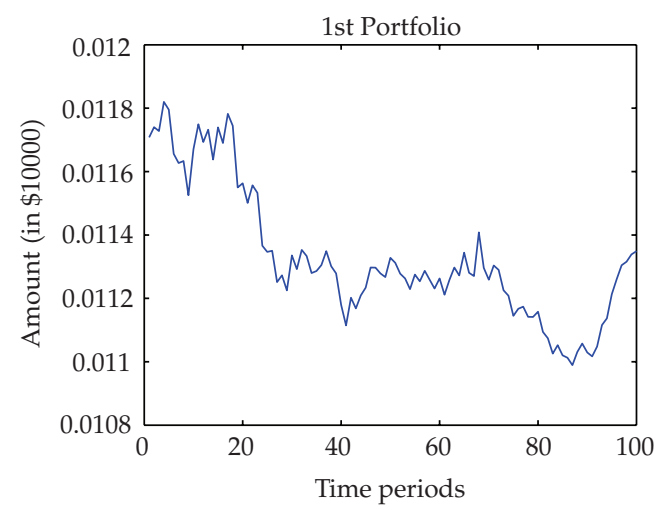

(a)

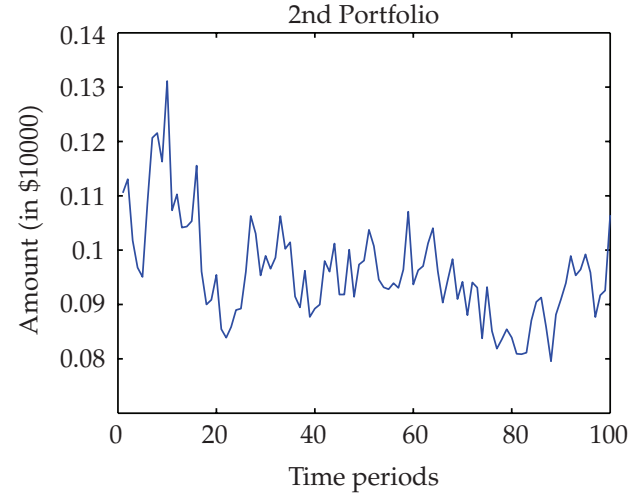

(b)

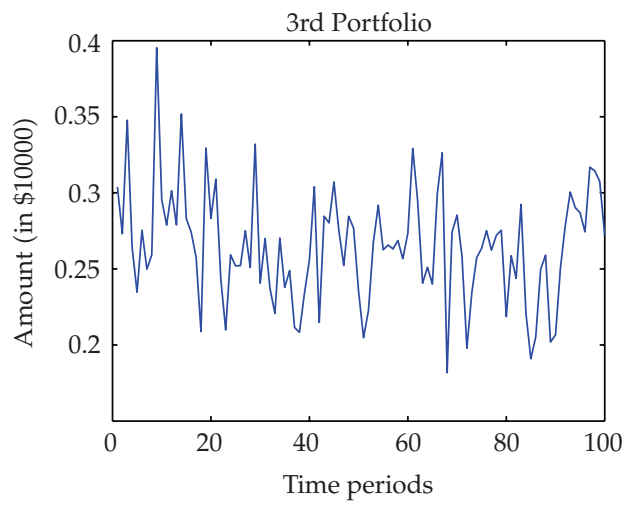

(c)

Figure 4: The evolution of the 1st, 2nd and 3rd classes of a heterogeneous Portfolio for the respective incidents, during 100 unit-time period.

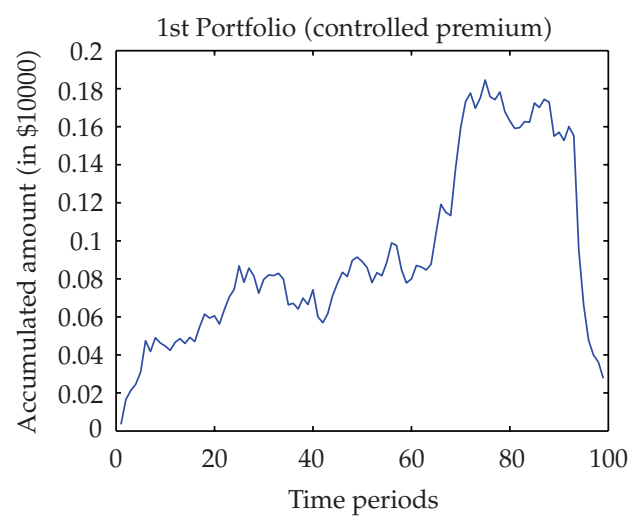

(a)

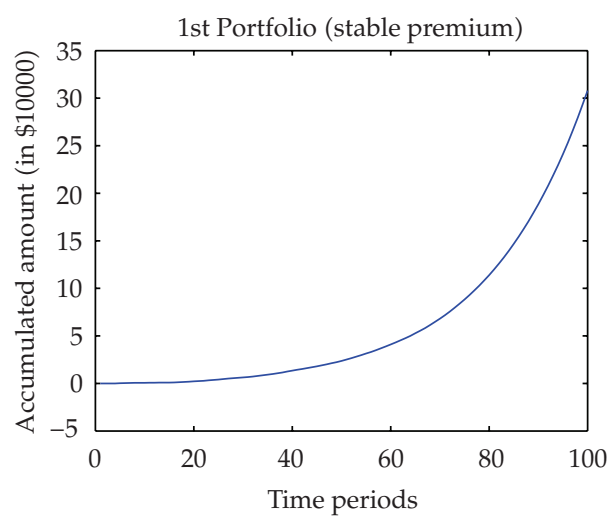

(b)

Figure 5: The accumulated profit/loss for (stochastic) controlled premium and stable premium, respectively, for the 1st class of a heterogeneous Portfolio for the respective incidents, during 100 unittime period (300000 simulations). 


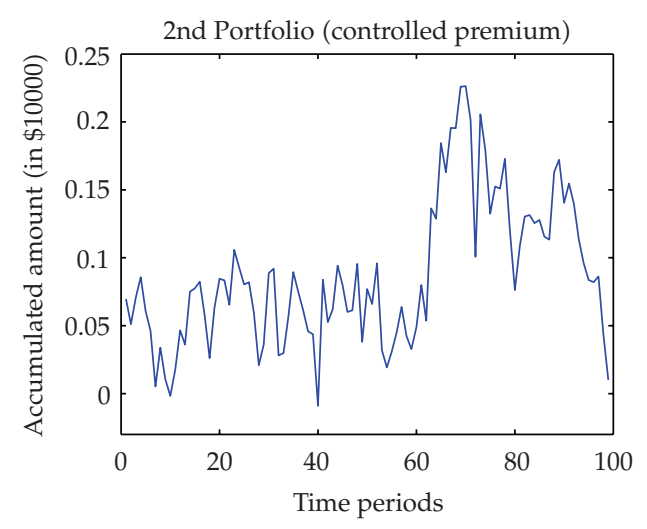

(a)

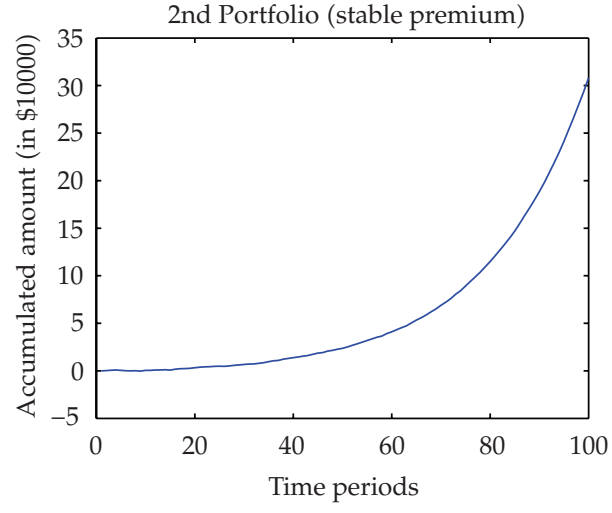

(b)

Figure 6: The accumulated profit/loss for (stochastic) controlled premium and stable premium, respectively, for the 2nd class of a heterogeneous Portfolio for the respective incidents, during 100 unittime period (300000 simulations).

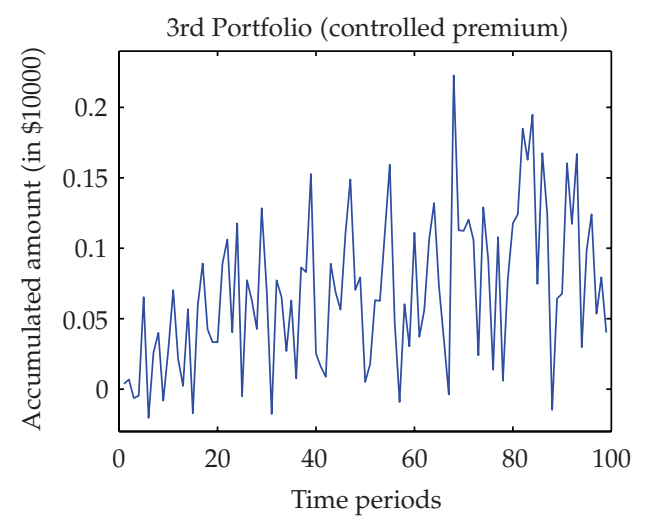

(a)

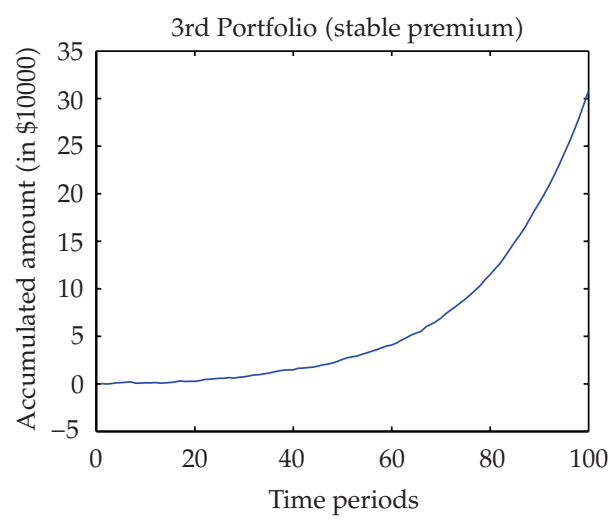

(b)

Figure 7: The accumulated profit/loss for (stochastic) controlled premium and stable premium, respectively, for the 3rd class of a heterogeneous Portfolio for the respective incidents, during 100 unittime period (300000 simulations).

$q_{i}$ is the probability of occurring the $i$ th -risk,

$\mu_{i}$ is the mean of the distribution of claims upon the $i$ th-risk occurs, and

$\sigma_{i}^{2}$ is the variance of the distribution of claims upon the $i$ th-risk occurs.

Then, the premium is calculated using the mean plus a fixed percentage of the variance of the risk. Of course, this approach results an explosive solvency margin as the additional safety loading is continuously accumulated over time.

In this paper, we introduced and developed a dynamic approach to the premium rating process for a heterogeneous portfolio of risks. The portfolio is divided into several classes where each class interacts to the others. Furthermore, the risks are modeled not statistically by distributions but dynamically by standard Brownian motions. The safety loading included in the premium is continuously revised using the volatility of the Brownian motion and the total accumulated solvency margin up to the specific time point. 
The resulting model exhibits two highly preferable features. Firstly, it has a balanced surplus development and not an explosive one till the end of the control period. Secondly, it concludes a much more competitive premium than the traditional approach.

Finally, we should stress three possible directions of further research. The first direction considers the same problem with a generalization as regards to the introduction of risky assets and consequently expansion of the number of the control variables. The second direction considers the substitution of the standard Brownian motions for the risks with fractional Brownian motions. Finally, the third direction considers the introduction of a non Markovian delay controller for smoothing the whole procedure. For those three projects, there is some research work in progress.

\section{Appendix}

\section{A. Stochastic Control Theory}

In this short appendix, we provide two basic theorems from Yong and Zhou [21] with respect to the necessary optimal control framework.

Theorem A.1. Let the linear quadratic stochastic control problem (A.1)-(A.2)

$$
\begin{gathered}
d \bar{\Pi}(t)=\{A(t) \bar{\Pi}(t)+\Sigma(t) \bar{\varepsilon}(t)\} d t-\Sigma(t) d \bar{W}(t), \\
\bar{\Pi}(0)=0, \\
J^{\bar{\varepsilon}}(t, \bar{\Pi})=\mathbb{E}^{t, \bar{\Pi}}\left\{\int_{0}^{T}\left[\bar{\Pi}^{\prime}(t) Q \bar{\Pi}(t)+\left(\bar{\varepsilon}(t)-\bar{\varepsilon}_{\tau}\right)^{\prime} R\left(\bar{\varepsilon}(t)-\bar{\varepsilon}_{\tau}\right)\right] d t+\bar{\Pi}^{\prime}(T) G \bar{\Pi}(T)\right\} .
\end{gathered}
$$

Then the optimal control for the $\bar{\varepsilon}^{*}(\cdot)$ vector is being described as a state feedback form

$$
\bar{\varepsilon}^{*}(t)=\bar{\varepsilon}_{\tau}-\Psi(t) \bar{\Pi}(t)-\psi(t) \quad \text { for } t \in[0, T]
$$

where

$$
\begin{gathered}
\Psi(t) \triangleq R^{-1} \Sigma^{\prime}(t) P(t), \quad B \Psi(t), D \Psi(t) \in L^{\infty}\left(0, T ; \mathbb{R}^{k \times k}\right), \\
\psi(t) \triangleq R^{-1} \Sigma^{\prime}(t) \phi(t), \quad B \psi(t), D \psi(t) \in L^{2}\left(0, T ; \mathbb{R}^{k}\right) .
\end{gathered}
$$

while $P(\cdot) \in C\left([0, T] ; \mathbb{R}^{k \times k}\right)$ is symmetric and $\phi(\cdot) \in C\left([0, T] ; \mathbb{R}^{k}\right)$ are obtained from the following matrix-stochastic equations:

$$
\begin{gathered}
\dot{P}(t)+P(t) A(t)+A^{\prime}(t) P(t)+Q(t)-P(t) \Sigma(t) R^{-1} \Sigma^{\prime}(t) P(t)=0, \\
P(T)=G, \quad \text { a.e. } t \in[0, T],
\end{gathered}
$$


and also

$$
\begin{gathered}
\dot{\phi}(t)+\left\{A(t)-\Sigma(t) R^{-1} \Sigma^{\prime}(t) P(t)\right\}^{\prime} \phi(t)=0, \\
\phi(t)=0, \quad \text { a.e.t } \in[0, T] .
\end{gathered}
$$

The proof of this theorem may be found in Yong and Zhou [21] work.

Moreover, the solution of (A.7) has the following form:

$$
\phi(t)=\zeta(t, 0) \phi_{0}
$$

we define

$$
\widetilde{A}(s)=\left[A(s)-\Sigma(s) R^{-1} \Sigma^{\prime}(s) P(s)\right]^{\prime} .
$$

Using Picard's successive approximation, the state transition matrix is given by the following expression, which is called the Peano-Baker series; see Antsaklis and Michel [22]:

$$
\zeta(t, 0)=I+\int_{0}^{t} \tilde{A}(s) d s+\int_{0}^{t} \tilde{A}\left(s_{1}\right) \int_{0}^{s_{1}} \tilde{A}\left(s_{2}\right) d s_{2} d s_{1}+\cdots+\int_{0}^{t} \tilde{A}\left(s_{1}\right) \cdots \int_{0}^{s_{n}} \tilde{A}\left(s_{n}\right) d s_{n} \cdots d s_{1}+\cdots
$$

However, it is profound that due to the terminal conditions $\phi(T)=0$ and $\zeta(T, 0) \neq 0$, we obtain $\phi_{0}=0$, which consequently gives $\psi(t) \triangleq 0$.

The proof for the following theorem may be also found in Yong and Zhou [21] work.

Theorem A.2. For $A(t), C(t) \in L^{\infty}\left(0, T ; \mathbb{R}^{k \times k}\right)$, and $b(t), d(t) \in L^{\infty}\left(0, T ; \mathbb{R}^{k}\right)$ one can consider the following linear stochastic differential equation:

$$
\begin{gathered}
d X(t)=\{A(t) X(t)+b(t)\} d t-\Sigma(t) d \bar{W}(t), \\
X(0)=0,
\end{gathered}
$$

and $\Phi(t)$ the solution of the following ordinary differential matrix equation:

$$
\begin{gathered}
d \Phi(t)=A(t) \Phi(t) d t, \\
\Phi(0)=I,
\end{gathered}
$$

and then the strong solution for $\mathrm{X}(\mathrm{t})$ of system (A.7) can be represented as

$$
X(t)=\Phi(t)\left\{\int_{0}^{t} \Phi(s)^{-1} b(s) d s+\int_{0}^{t} \Phi(s)^{-1} \Sigma(s) d \bar{W}(s)\right\}
$$


where also

$$
\begin{gathered}
d\left(\Phi(t)^{-1}\right)=-\Phi(t)^{-1} A(t) d t \\
\Phi(0)^{-1}=I
\end{gathered}
$$

Thus, the solution of the (nonhomogeneous) linear stochastic differential equation is given by the following expression:

$$
\bar{\Pi}(t)=\Phi(t)\left\{\int_{0}^{t} \Phi(s)^{-1} b(s) d s+\int_{0}^{t} \Phi(s)^{-1} \Sigma(s) d \bar{W}(s)\right\}
$$

\section{Acknowledgment}

This work was supported by the reinforcement program of Human Research Manpower "PENED" in the framework of Measure 8.3, Action 8.3.1 of the operational program of competitiveness, Third Community Support Program.

\section{References}

[1] N. L. Bowers, H. U. Gerber, J. C. Hickman, D. A. Jones, and C. Nesbitt, Journal of Actuarial Mathematics, 1997, The Society of Actuaries.

[2] Y. Zaks, E. Frostig, and B. Levikson, "Optimal pricing of a heterogeneous portfolio for a given risk level," ASTIN Bulletin, vol. 36, no. 1, pp. 161-185, 2006.

[3] A. Tustin, The Mechanism of Economic Systems: An Approach to the Problem of Economic Stabilisation from the Point of View of Control-System Engineering, Harvard University Press, Cambridge, Mass, USA, 1953.

[4] S. Holly and H. A. Hallett, Optimal Control, Expectations and Uncertainty, Cambridge University Press, Cambridge, UK, 1989.

[5] K. Borch, "Die optimale Dividendenpolitik der Unternehmen," Recherche Opérationnelle, vol. 11, no. 1, pp. 131-143, 1967.

[6] A. Martin-löf, "Lectures on the use of control theory in insurance," Scandinavian Actuarial Journal, pp. $1-25,1994$.

[7] S. Asmussen and M. Taksar, "Controlled diffusion models for optimal dividend pay-out," Insurance: Mathematics and Economics, vol. 20, no. 1, pp. 1-15, 1997.

[8] M. Schäl, "On piecewise deterministic Markov control processes: control of jumps and of risk processes in insurance," Insurance: Mathematics and Economics, vol. 22, no. 1, pp. 75-91, 1998.

[9] B. Højgaard and M. Taksar, "Controlling risk exposure and dividends payout schemes: insurance company example," Mathematical Finance, vol. 9, no. 2, pp. 153-182, 1999.

[10] M. I. Taksar, "Optimal risk and dividend distribution control models for an insurance company," Mathematical Methods of Operations Research, vol. 51, no. 1, pp. 1-42, 2000.

[11] C. Hipp and M. Taksar, "Stochastic control for optimal new business," Insurance: Mathematics and Economics, vol. 26, no. 2-3, pp. 185-192, 2000.

[12] C. Irgens and J. Paulsen, "Optimal control of risk exposure, reinsurance and investments for insurance portfolios," Insurance: Mathematics and Economics, vol. 35, no. 1, pp. 21-51, 2004.

[13] H. Yang and L. Zhang, "Optimal investment for insurer with jump-diffusion risk process," Insurance: Mathematics and Economics, vol. 37, no. 3, pp. 615-634, 2005.

[14] W. M. Wonham, "On a matrix Riccati equation of stochastic control," SIAM Journal of Control and Optimization, vol. 6, no. 4, pp. 681-697, 111968.

[15] P. McLane, "Optimal stochastic control of linear systems with state- and control-dependent disturbances," IEEE Transactions on Automatic Control, vol. 16, no. 6, pp. 793-798, 1971.

[16] M. H. A. Davis, Linear Estimation and Stochastic Control, Chapman and Hall, London, UK, 1977. 
[17] A. Ichikawa, "Dynamic programming approach to stochastic evolution equations," SIAM Journal on Control and Optimization, vol. 17, no. 1, pp. 152-174, 1979.

[18] S. Chen and J. Yong, "Stochastic linear quadratic optimal control problems," Applied Mathematics and Optimization, vol. 43, no. 1, pp. 21-45, 2001.

[19] I. G. Petrovsky, Lectures on theory of ordinary differential equations, Nauka, Moscow, Russia, 1970.

[20] P. E. Kloeden and E. Platen, Numerical Solution of Stochastic Differential Equations, Springer, Berlin, Germany, 1992.

[21] J. Yong and X. Y. Zhou, Stochastic Controls, Springer, New York, NY, USA, 1999.

[22] P. J. Antsaklis and A. N. Michel, Linear Systems, The McGraw-Hill, Columbus, Ohio, USA, 1997. 


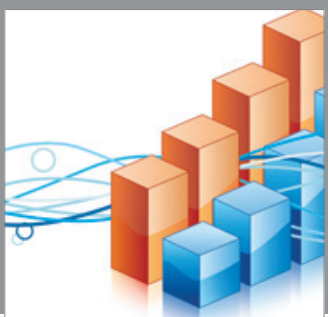

Advances in

Operations Research

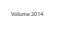

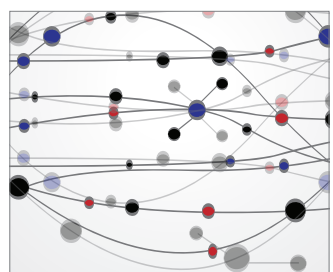

\section{The Scientific} World Journal
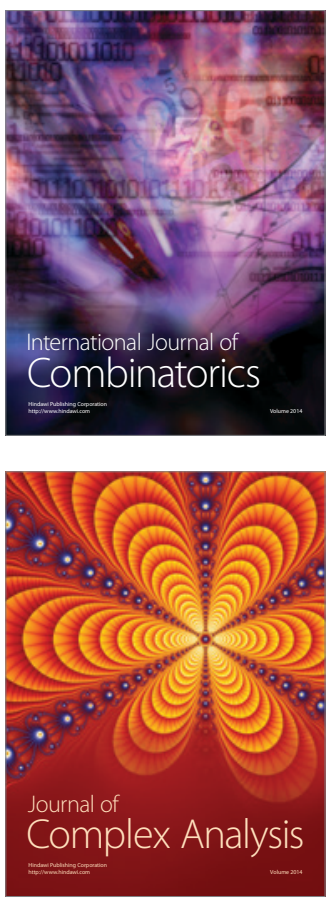

International Journal of

Mathematics and

Mathematical

Sciences
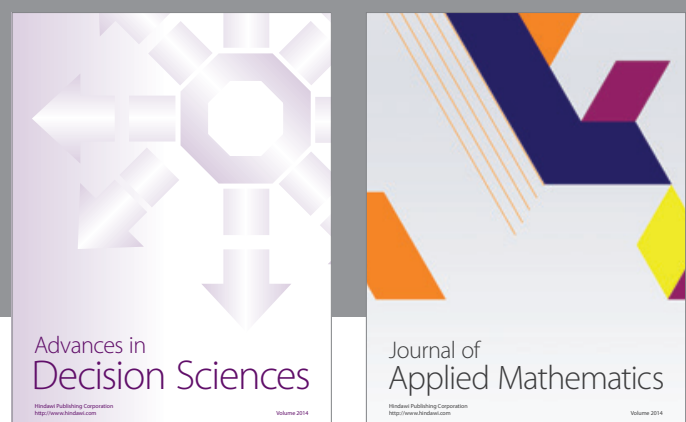

Journal of

Applied Mathematics
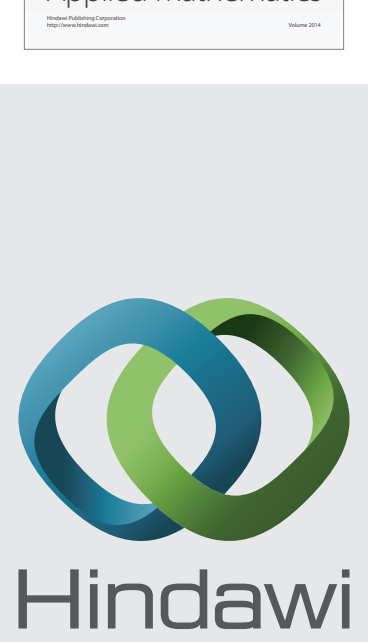

Submit your manuscripts at http://www.hindawi.com
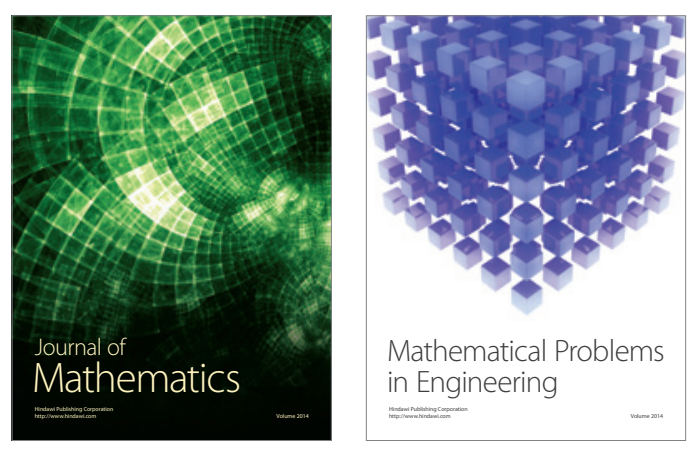

Mathematical Problems in Engineering
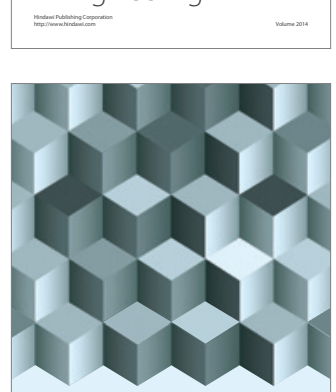

Journal of

Function Spaces
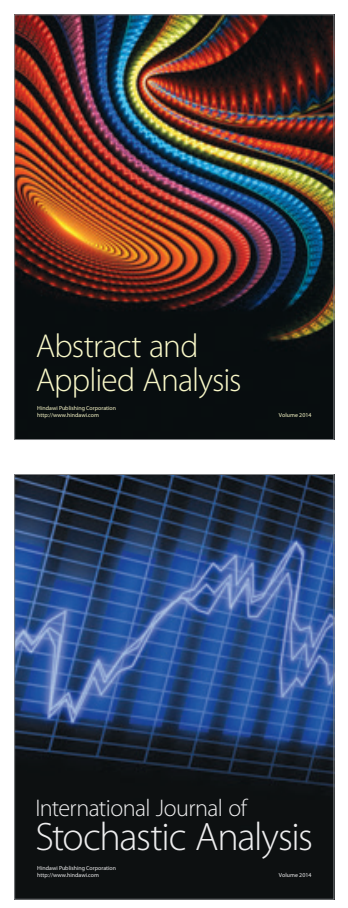

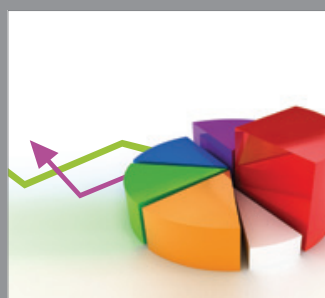

ournal of

Probability and Statistics

Promensencen
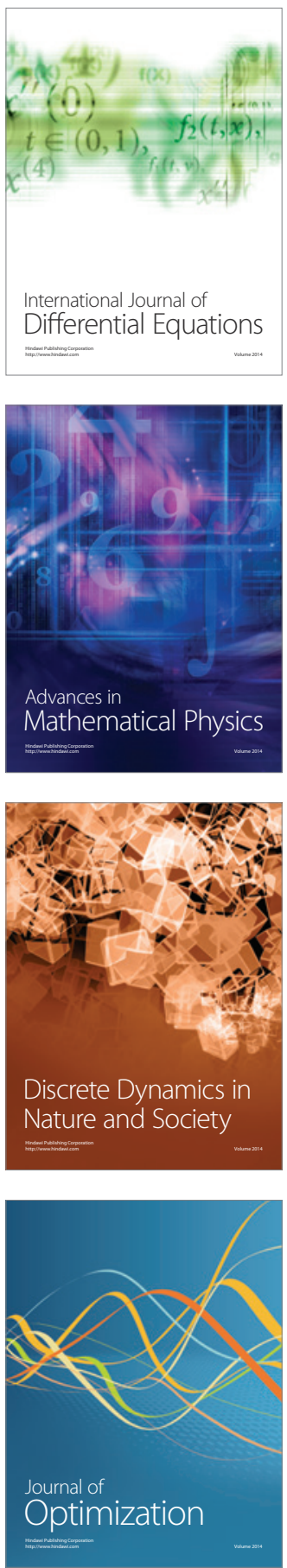